confermati dalla diagnosi emoscopica. Tutti gli altri 52 campioni risultavano negativi con entrambi i metodi.

Conclusioni I risultati ottenuti dimostrano l'utilità e l'efficacia del test immunocromatografico.

Va in ogni caso ricordato che all'impiego del test rapido deve essere sempre associato l'esame emoscopico, sia per la ricerca (parassitemie inferiori a $0,002 \%$ ) e la diagnosi di specie, sia per la determinazione dell'indice di parassitemia.

\title{
DIAGNOSTICA DELLA MALARIA: VALIDITÀ ED UTILITÀ DEL TEST RAPIDO IMMUNOCROMATOGRAFICO
}

\author{
Menghini L.G., Sartori R., Ober P., lole C., Caciagli P. \\ Laboratorio di Microbiologia Ospedale di Trento
}

Introduzione L'identificazione del parassita malarico viene comunemente effettuata su preparati microscopici di sangue periferico: lo striscio sottile e la goccia spessa. La diagnosi emoscopica, per la sua semplicità, economicità e rapidità di risposta, rappresenta ancora il metodo standard per la diagnosi di malaria, tuttavia da diversi anni sono disponibili altre tecniche, alcune delle quali standardizzate in kit commerciali, che hanno lo scopo di rendere più semplice e rapida la diagnosi.

Lo studio si propone di valutare l'utilità del test rapido immunocromatografico come ausilio alla diagnosi emoscopica di malaria.

Metodi Nel periodo giugno 2002-maggio 2005 sono stati testati 66 campioni di sangue intero da pazienti che presentavano un quadro clinico anamnestico sospetto per infezione malarica (febbre e recente viaggio in Paesi endemici per malaria). Il $62,1 \%$ dei soggetti proveniva dall'Africa (78\% di nazionalità italiana), il 19,7\% da Paesi asiatici (76,9\% italiani) ed il restante $18,2 \%$ dall'America Latina (100\% italiani). Dei 66 soggetti oltre $1^{\prime} 80 \%$ vi si era recata per motivi di turismo e/o lavoro. Tutti i pazienti esaminati non avevano effettuato la chemioprofilassi antimalarica. La ricerca dei plasmodi è stata eseguita contemporaneamente con esame microscopico (striscio sottile e goccia spessa) e test immunocromatografico (OptiMAL Rapid Malaria Test della DiaMed). La metodica si basa sull'individuazione nel sangue intero dell'enzima lattato deidrogenasi ( $\mathrm{pLDH})$ e riconosce sia Plasmodium falciparum sia, genericamente, gli altri plasmodi (Plasmodium spp.).

Risultati Il test immunocromatografico è risultato positivo in 10 casi $(71,43 \%), 7$ per $P$. falciparum e 2 per Plasmodium spp., debolmente positivo in 3 casi per $P$. falciparum e in un caso per Plasmodium spp. $(28,57 \%)$. I casi sono stati tutti 